\title{
Principles of progenitor temporal patterning in the developing invertebrate and vertebrate nervous system Polina Oberst ${ }^{1,4}$, Gulistan Agirman ${ }^{1,2,4}$ and Denis Jabaudon ${ }^{1,3}$
}

During the development of the central nervous system, progenitors successively generate distinct types of neurons which assemble into the circuits that underlie our ability to interact with the environment. Spatial and temporal patterning mechanisms are partially evolutionarily conserved processes that allow generation of neuronal diversity from a limited set of progenitors. Here, we review examples of temporal patterning in neuronal progenitors in the Drosophila ventral nerve cord and in the mammalian cerebral cortex. We discuss cell-

autonomous mechanisms and environmental influences on the temporal transitions of neuronal progenitors. Identifying the principles controlling the temporal specification of progenitors across species, as highlighted here, may help understand the evolutionary constraints over brain circuit design and function.

\footnotetext{
Addresses

${ }^{1}$ Department of Basic Neurosciences, University of Geneva, Switzerland ${ }^{2}$ GIGA-Neurosciences, University of Liège, C.H.U. Sart-Tilman, Liège, Belgium

${ }^{3}$ Department of Neurology, Geneva University Hospital, Geneva, Switzerland

Corresponding author: Jabaudon, Denis (denis.jabaudon@unige.ch) ${ }^{4}$ Contributed equally to this work.
}

\author{
Current Opinion in Neurobiology 2019, 56:185-193 \\ This review comes from a themed issue on Neuronal identity \\ Edited by Sacha Nelson and Oliver Hobert \\ For a complete overview see the Issue and the Editorial \\ Available online 15th April 2019 \\ https://doi.org/10.1016/j.conb.2019.03.004 \\ 0959-4388/@ 2019 Elsevier Ltd. All rights reserved.
}

\section{Introduction}

Neurons are the building blocks of the circuits of the central nervous system (CNS). As such, initial neuronal diversity sets the frame for the diversity of circuits that can be built, and hence for an animal's behavioral repertoire. The last few years have provided us with an increasingly detailed census of the distinct neuronal cell types that populate the CNS, and particularly the cerebral cortex, thanks in particular to the advent of high-throughput single-cell technologies (reviewed in Ref. [1]). Despite this expanded cellular taxonomy, the origins of neuronal diversity remain poorly understood.
Two main processes have been involved in the developmental generation of diverse types of cells of the CNS: spatial patterning and temporal patterning. The patterning of molecularly distinct progenitors and daughter cells into separate spatial domains ('spatial patterning') is widespread throughout the CNS, including in the retina [2], spinal cord [3], cerebellum [4], and ventral pallium [5]. In addition to this process, however, in many cases, neuronal diversity also emerges from 'temporal patterning', that is, in the successive emergence of progenitors and neurons with distinct molecular properties within confined brain regions. In this review, we will focus on this latter process and highlight select aspects of temporal progression in neural progenitor identity and their ability to sequentially generate diverse neuronal subtypes in the developing Drosophila ventral nerve cord (VNC) and mouse neocortex. Our review is focused on select examples of temporal patterning in both of these species, and interested readers can refer to previous reviews addressing other aspects of nervous system patterning (in particular spatial patterning) [6-9].

\section{Temporal patterning in Drosophila neuroblasts}

Much of our understanding of the mechanisms controlling neuronal specification by temporal patterning comes from studies performed in the common fruit fly Drosophila melanogaster. Temporal patterning in Drosophila occurs throughout the developing CNS (comprising the central brain, the optic lobes, and the VNC) during both embryonic and larval stages. Here, we review select examples of temporal patterning in the embryonic $\mathrm{VNC}$, since exhaustive reviews on the temporal specification in the Drosophila CNS across developmental stages have recently been published [10-12].

Neurons and glial cells of the Drosophila VNC are generated by neural stem cells called neuroblasts. Each neuroblast has a unique spatial identity (resulting in around 100 unique neuroblasts in each lobe of the central brain, and around 30 unique neuroblasts in each hemisegment of the VNG (reviewed in Ref. [6]) and produces a stereotyped series of progeny over time [13]. Drosophila neurogenesis occurs in two sequential waves; a first wave occurs during embryogenesis (contributing to around $10 \%$ of adult neurons), which is followed by a longer second wave spanning larval and pupal stages, during which the vast majority of neurons and glia of the adult CNS is generated [14,15] (Figure 1a). 
Figure 1

(a)

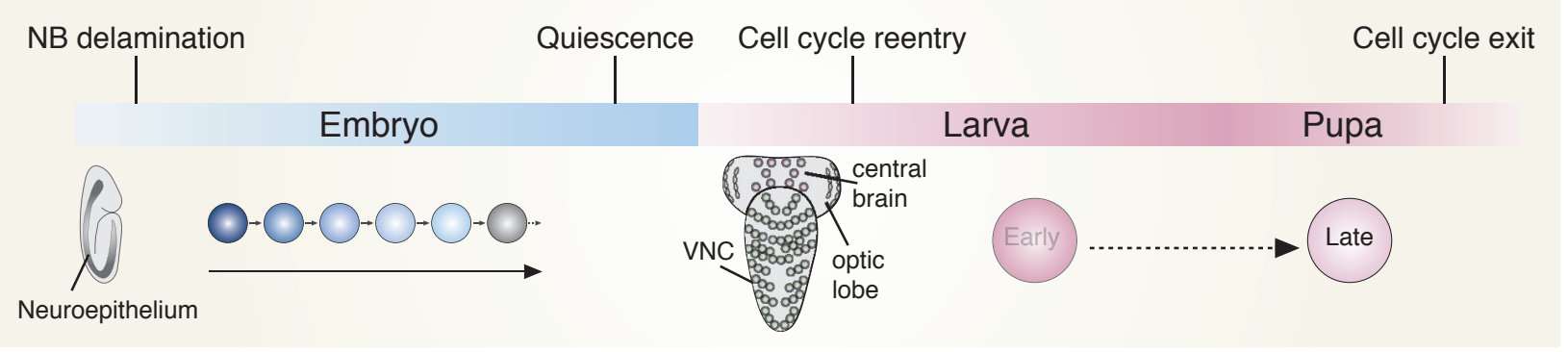

(b)

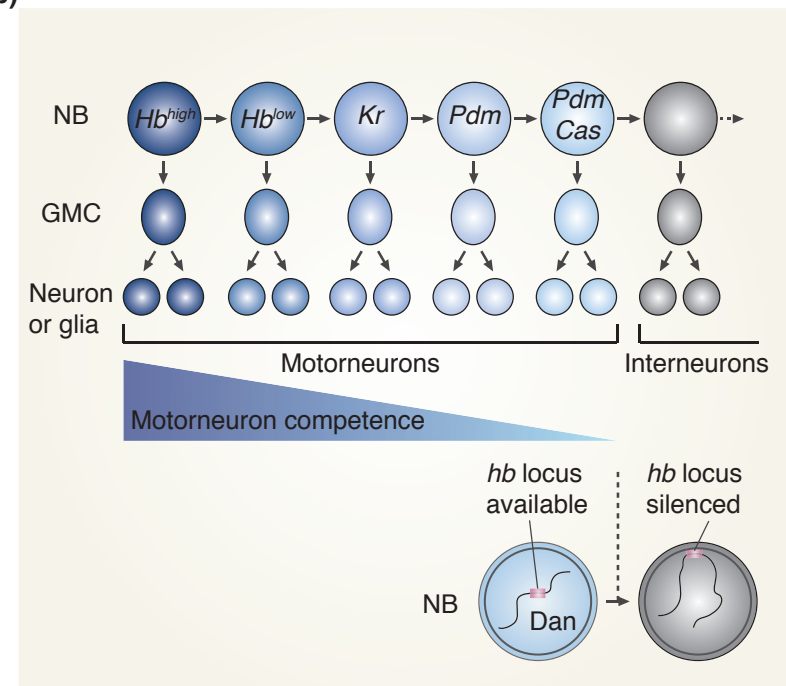

(c)

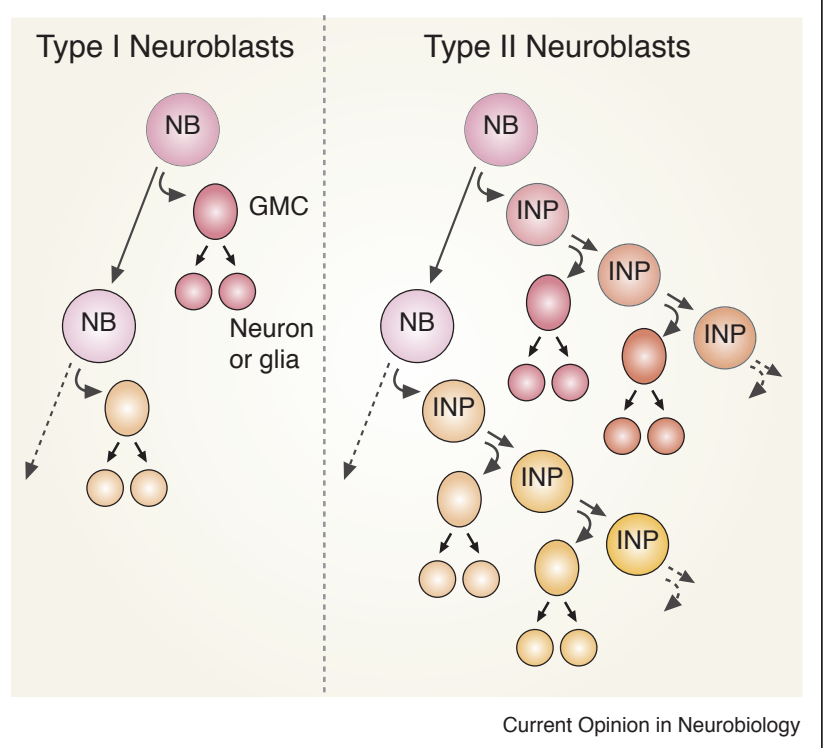

Neural development and temporal patterning in Drosophila.

(a) Neuroblasts delaminate from the neuroepithelium early in embryogenesis and pass through sequential temporal states to generate different types of neurons before entering quiescence at the end of embryogenesis. During larval stages, neuroblasts re-enter the cell cycle and pass through additional temporal states to generate distinct types of neurons. Terminal cell cycle exit occurs during pupal stages. (b) Embryonic VNC neuroblasts transition through a neuroblast-intrinsic tTF cascade and give rise to distinct neurons during each temporal window. In the NB7-1 and NB3-1 lineages, neuroblasts first generate five distinct motorneurons and then switch to producing interneurons. Competence to generate motorneurons is lost at the transition to interneuron generation through epigenetic silencing of developmental genes, here exemplified by the silencing of the $h b$ locus in the absence of the neuroblast nuclear protein Dan. (c) Division modes of neuroblasts in the larval central brain. Type I neuroblasts divide asymmetrically and produce a GMC at each division, which divides once more to generate a pair of neurons or glia. Type II neuroblasts give rise to INPs, which progress through their own temporal series and divide several times to give rise to distinct GMCs at each division, generating an additional layer of neuronal diversity.

Abbreviations: GMC, ganglion mother cell; Hb, Hunchback; INP, intermediate neural progenitor; NB, neuroblast; tTF, temporal transcription factor; VNC, ventral nerve cord.

Temporal patterning has been first and best described in Drosophila embryonic VNC [16-18]. The VNC contains type I neuroblasts, which have short lineages and divide a total of about five times, within a single day. Type I neuroblasts generate a neuroblast and a ganglion mother cell (GMC) at each division; the latter divides once more to produce a pair of neurons or glial cells. Early-born progeny are displaced by later-born progeny, resulting in a laminar organization of the $\mathrm{VNC}$ reflecting birth order: early-born neurons are located in deep layers and late-born neurons in more superficial layers [2], as is the case in the mammalian cerebral cortex $[19,20]$.

Each neuroblast in the embryonic VNC sequentially expresses a series of temporal transcription factors ( $\mathrm{tTFs}$ ), that is, transcription factors which specify the temporal identity of neurons born during their time window of expression. Hunchback (Hb) is expressed first, followed by Kruppel (Kr), POU domain proteins 1 and 2 (Pdm), Castor and Grainy head [16-18] (Figure 1b). tTF 
expression progression occurs at approximately each neuroblast division and the same series of tTFs is active in different neuroblast lineages, in each of which it specifies distinct neuronal fates. Thus, the same tTF can specify an interneuron in one lineage, and a motor neuron in another [2,17]. As will be discussed below, some Drosophila tTF have mammalian orthologs (e.g. Hunchback Ikzf1 (Ikaros) and Castor - Casz1), which appear to also be involved in the progression of temporal identity in retinal and cortical progenitors [21-23]. Interestingly, daughter neurons continue to express the tTF that their mother cell was expressing, although the role of this expression is unknown [2].

Of note, the Drosophila central brain contains an additional, less abundant type of neuroblast (type II neuroblasts), which gives rise to transit amplifying intermediate neural progenitors (INPs) with a limited proliferative capacity [24,25]. INPs progress through their own series of t'TFs (Dichaete $\rightarrow$ Grainy head $\rightarrow$ Eyeless) and specify distinct cell types during each temporal window. Thus, in the Drosophila central brain, the two parallel axes of temporal progression in type II neuroblasts and INPs combinatorially specify cell identities and increase neuronal diversity [26] (Figure 1c).

\section{Control of temporal transitions}

How is the successive expression of tTFs regulated? Studies in embryonic VNC neuroblasts suggest that transcriptional cross-regulation between tTFs together with additional independent mechanisms act to regulate temporal transitions [2,16-18,27]. Prolonged expression of the early-onset $\mathrm{t}$ TFs $\mathrm{Hb}$ or $\mathrm{Kr}$ blocks the progression of neuroblast neurogenic competence and results in the excessive production of early-born neurons at the expense of later-born ones [17,27]. $\mathrm{Hb}$ and $\mathrm{Kr}$ promote expression of the following $\mathrm{t} T \mathrm{TF}$ in the series and repress the next-plus-one factor [17], but removal of $\mathrm{Hb}$ or $\mathrm{Kr}$ leads to loss of only one temporal identity window, without affecting subsequent temporal transitions $[2,17]$. This suggests that t'TFs do not alone account for temporal transitions, but that other factors, possibly including extrinsic signaling as occurs in other parts of the nervous system (see below), are at play. Transition from $\mathrm{Hb}$ to $\mathrm{Kr}$ expression requires repression of $\mathrm{Hb}$ through the orphan nuclear receptor Svp. Translation of Svp protein is coupled to cytokinesis, and thus the transition from $\mathrm{Hb}$ to $\mathrm{Kr}$ requires cell division [27-30]. Interestingly, the mammalian Svp homologs COUP-TF1/2 (Nr2f1 and $N r 2 f 2$ ) act in mammalian cortical progenitors to promote the switch from early-born to late-born neuron production, and from neurogenesis to gliogenesis [31], suggesting an evolutionarily conserved role in regulating temporal transitions. All other transitions examined, however, have been shown to occur even in G2-arrested neuroblasts [27], as also seems to be the case for cellcycle arrested mammalian cortical progenitors [32 $2^{\bullet \bullet}$, such that 'counting' of cell divisions does not seem to be an obligatory process for temporal progression in identity. Finally, clonally cultured VNC neuroblasts progress normally through the temporal TF cascade, suggesting that lineage-intrinsic cues are sufficient to mediate temporal progression [16,27], although feedback cues from neural progeny might play a role. Of note, extrinsic factors have been implicated in the temporal progression of larval central brain type II neuroblasts: for example, ecdysone signaling via the EcR-B1 receptor initiates a major earlyto-late gene expression transition, and lack of this signaling leads to maintained expression of early temporal factors $\left[33^{\bullet \bullet}, 34\right]$.

\section{Temporal plasticity of Drosophila neuroblast competence}

The competence of neuroblasts to successively produce distinct neuronal types at successive stages of their lineage has been best studied using ectopic (i.e. heterochronic) expression of t'TFs $[35,36]$. Ectopic expression of the early $\mathrm{t} \mathrm{TF} \mathrm{Hb}$ at later stages in the NB7-1 neuroblast lineage induces the generation of early-born neuronal types that are normally specified during the $\mathrm{Hb}$ expression window $[35,36]$. However, competence to respond to ectopic $\mathrm{Hb}$ is lost after the fifth division of this neuroblast, at a time point when daughter cell fate switches from motor neurons to interneurons. This loss of competence to respond to $\mathrm{Hb}$ is thought to be due to a repositioning of $\mathrm{Hb}$ target genes close to the nuclear lamina, which renders them inaccessible, as exemplified by the silencing of $H b$ itself through such a process [37] (Figure 1b). This genomic reorganization occurs in near synchrony within the entire neuroblast population, suggesting that an extrinsic global signal may trigger this process [2,37]. Similarly, in the NB7-1 and NB3-1 lineages, $\mathrm{Kr}$ specifies third-born U3 motor neurons and its mis-expression between the third and fifth neuroblast division induces the generation of such motor neurons [36], but the competence to respond to $\mathrm{Kr}$ is lost when the neuroblast transits to generating interneurons. Polycomb repressive complexes (PRCs), which are multi-protein complexes that inhibit transcription via epigenetic silencing, restrict the competence of NB7-1 and NB3-1 neuroblasts to respond to $\mathrm{Kr}$, such that decreased $\mathrm{PRC}$ activity extends the competence window for motor neuron generation [38]. PRCs are also found in mammalian neural progenitors, where they regulate progenitor identity and control the switch from neurogenesis to gliogenesis [39,40]. Finally, progressive restriction in the competence to respond to specific signals is not limited to neuroblasts but has also been observed in intermediate progenitors, which lose competence to respond to Notch-signaling as they age [41].

\section{Temporal patterning in the mammalian cerebral cortex}

As is the case in Drosophila, at least some neural progenitors in vertebrates also generate distinct neuronal subtypes over time, and this process has been particularly 
well studied in the mouse neocortex. The neocortex is organized in six layers, each enriched in specific subtypes of neurons with distinct molecular identities, morphologies, and connectivity [19,20]. In the developing neocortex, excitatory neurons are generated from apical progenitors (APs) located in a deep germinal zone adjacent to the lateral ventricles (ventricular zone, VZ). From E11.5 to E16.5, APs divide to self-renew and to produce daughter neurons and daughter intermediate progenitors (IPs, also called basal progenitors). The latter cells move away from the ventricular zone to form a second germinal zone (subventricular zone, SVZ) and undergo only a few rounds of neurogenic divisions. Cortical neurons can thus be born directly from APs or indirectly from IPs, and laminarly distinct subtypes of neurons are sequentially generated from these cells, with deep-layer neurons being born first and superficial layer neurons last, as is the case in Drosophila VNC [2] (Figure 2a). Toward the end of the neurogenic period, around E17.5, APs undergo terminal divisions to generate glial cells $[19,42]$. The competence of APs to generate temporally defined daughter cell types results from the interplay of both cell-autonomous mechanisms and local and long-range environmental cues. The transcriptional, environmental, and epigenetic influences on APs temporal patterning are described in the following sections.

\section{AP neurogenic competence across neurogenesis}

Clonal analysis of E12.5 APs using the Mosaic Analysis with Double Markers (MADM) technology has shown that the majority of APs produce approximately 8-9 neurons (range from 3 to 16) that settle in both deep and superficial layers [42]. This indicates that as is the case for Drosophila neuroblasts, APs progressively acquire competence to generate distinct neuronal subtypes. Supporting these observations, in vivo genetic fate mapping of early APs expressing the deep layer marker FEZF2 showed that these progenitors exist throughout corticogenesis and sequentially generate deep then superficial layer projection neurons [43]. Subsets of fate-restricted progenitors may, however, exist, since CUX2-expressing progenitors have been proposed to exclusively produce superficial layer neurons. These cells were found in the ventricular zone as early as E12.5, and would undergo several rounds of proliferative divisions before undergoing neurogenic divisions at the time of superficial layer neuron generation [44]. Such fate-restricted progenitors, if present at all, are probably rare, however, since they have not yet conclusively been identified in single-cell RNA sequencing datasets $[45,46]$.

Cortical progenitors cultured in vitro recapitulate the normal course of corticogenesis and produce early-born deep layer neurons before generating late-born superficial layer neurons [47]. However, several studies have reported an underrepresentation of late-born superficial layer neurons in in vitro systems [47,48], and the proportion of early-born to late-born fates is influenced by culture conditions $\left[32^{\bullet \bullet}, 49\right]$. Supporting these findings, gene expression studies have shown that while progenitors cultured as aggregates or organoids are progressively changing their temporal gene expression profile over time, isolated progenitors where cell-cell contacts are prevented show only limited progression of temporal gene expression $\left[32^{\bullet \bullet}, 49\right]$. Similarly, while only a limited number of late-born neurons was detected in an in vitro system using human embryonic stem cell-derived cortical progenitor cells, transplantation into a neonatal mouse significantly increased the production of late-born superficial layer neurons [48]. Together, these findings suggest that temporal progression of cortical progenitors may require additional cell-extrinsic cues to express their competence to generate superficial layer neurons.

An important question is whether fate progression of cortical progenitors necessarily implies fate restriction. Spatially parcellated progenitors belong to distinct lineages and are thus relatively independent in their fate progression. Temporal parcellation instead requires a mechanism to repress past competences and induce new ones, but whether this mechanism is reversible or not has not been systematically examined with modern analytic tools. Seminal work in the ferret has investigated the plasticity in the neurogenic competence of cortical progenitors at different developmental stages using heterochronic transplantations. These studies revealed that early progenitors transplanted into a late environment are able to produce late-born superficial layer neurons, suggesting that early progenitors are multipotent and respond to cues present in a later environment [50,51]. In contrast, late progenitors transplanted into younger hosts did not reset their neurogenic competence and invariably gave rise to superficial layer neurons [52], suggesting that progenitors at late stages of corticogenesis become fate-restricted. A different interpretation, however, is that at late stages of corticogenesis, and particularly in the ferret, transplanted progenitors consist mostly of transit amplifying cells, including IPs, rather than APs. Thus, as suggested by a recent preprint from our laboratory, the lack of plasticity in neurogenic competence upon transplantation into younger hosts may reflect cell-type specific differences in AP and IP competence rather than a progressive restriction in the competence of APs [53].

\section{Control of temporal transitions in APs}

In contrast to the well-characterized temporal sequence of tTFs in Drosophila, the temporal transcriptional dynamics in mammalian cortical progenitors are still relatively poorly described with only few genes identified so far. Cortical progenitors express the transcription factor 
Figure 2

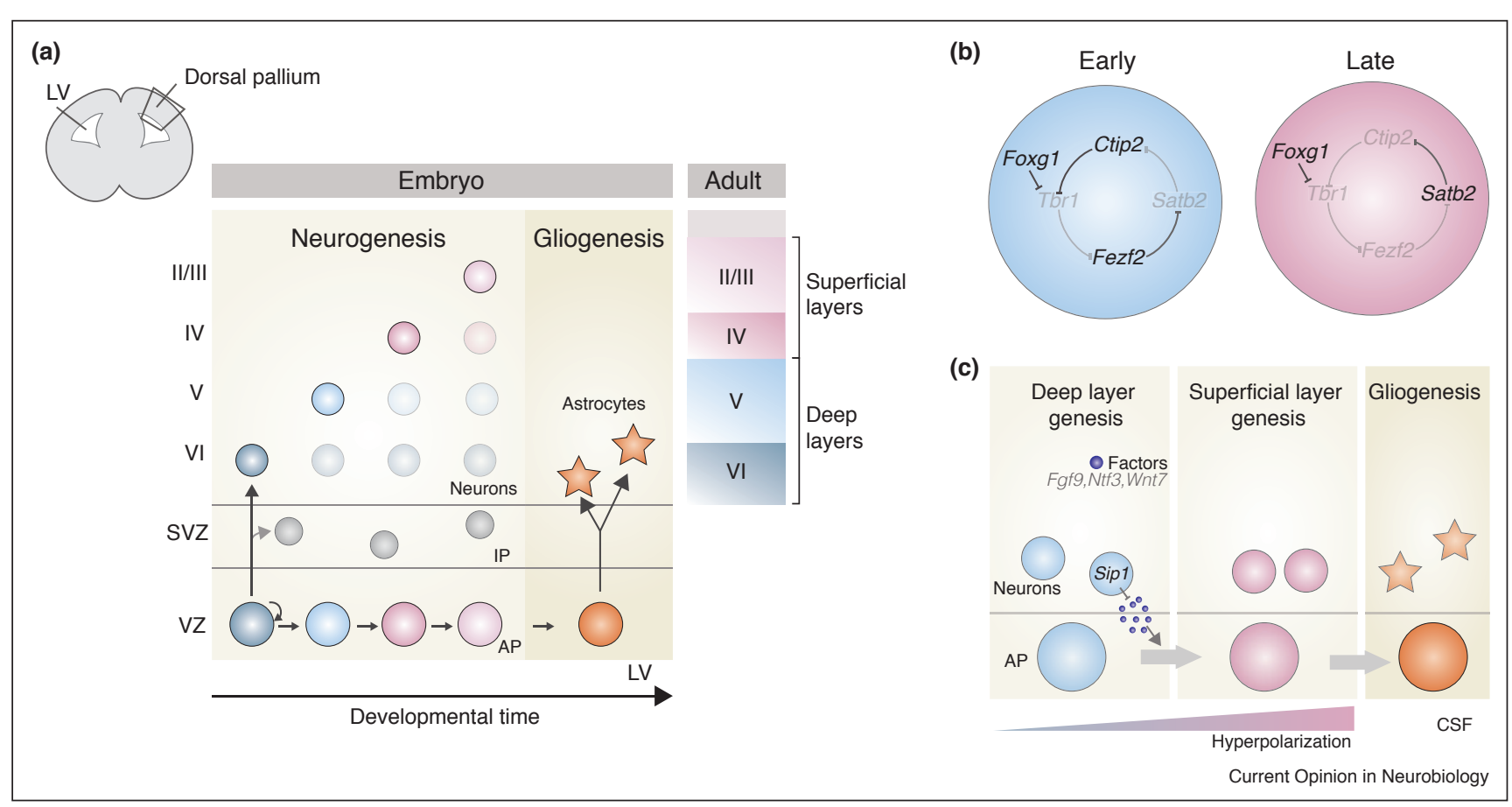

Overview of the mammalian cortical development.

(a) In the developing dorsal pallium, excitatory glutamatergic neurons born directly from APs or indirectly from IPs are generated in sequential waves that organize one above the other and form six distinct neuronal layers in the adult neocortex. At late corticogenesis, APs undergo selfconsuming symmetric division to generate glial cells. (b) Cross-talk between a core transcription factor network regulating deep versus superficial layer identity. Ctip2 and Fezf2 instruct deep layer identity and are expressed at early corticogenesis. Later in development, Satb2 expression is triggered and instruct superficial layer neuron identity. (c) Illustration of intrinsic and extrinsic influences on the temporal transitions of APs. Signaling factors from postmitotic neurons feedback to APs and instruct the transition from deep layer to superficial layer genesis. Progressive hyperpolarization of APs membrane potential constitutes an additional mechanism of temporal regulation. Finally, dynamic changes in the composition of the CSF could modulate APs behavior throughout corticogenesis.

Abbreviations: AP, apical progenitors; CSF, cerebrospinal fluid; IP, intermediate progenitors; LV, lateral ventricles; SVZ, subventricular zone; VZ, ventricular zone.

FOXG1 during early stages of corticogenesis, as they transit from Cajal Retzius cell production to deep layer neuron production. Loss of FOXG1 at mid stages of corticogenesis leads to heterochronic generation of Cajal Retzius neurons, thus suggesting that continued FOXG1 expression is necessary to suppress Cajal Retzius production [54]. A core transcriptional network of layer-enriched transcription factors including FEZF2, CTIP2, TBR1 and SATB2 has been identified over recent years [55]. These factors cross-regulate each other's expression, which is thought to allow the sequential acquisition of deep then superficial layer identities in newborn neurons (Figure 2b). However, none of these transcription factors are clearly temporally regulated and some of them (e.g. CTIP2) are expressed in post-mitotic neurons but not in APs [56-59]. Interestingly, late-born neurons initially express a combination of lamina-specific markers, and only later their identity is refined to include only superficial neuron markers. This process, which has been termed 'transcriptional priming' and which is also found in the hematopoietic system, suggests that final neuronal identity is progressively acquired in the course of development $\left[46,60^{\circ}, 61\right]$.

Despite the lack of unequivocal tTFs in the neocortex, the mammalian homolog of Hunchback Ikaros (Ik\&f1) provides a potential example of evolutionary functional conservation, as it is highly expressed in APs during early corticogenesis and promotes early-born deep layer fates [22]. Induction of Ikaros expression in late cortical progenitors (where it is normally downregulated) is not, however, sufficient to induce ectopic generation of deep layer neurons. This suggests that competence to respond to Ikaros is lost over time, reminiscent of the progressive loss of competence to respond to Hunchback and Kruppel in Drosophila neuroblasts [35-38]. FEZF2, which is expressed by early-born, deep-layer neurons is able to give rise to such neurons when overexpressed later in corticogenesis, but cannot per se be called a tTF, since the progeny of FEZF2 expressing APs are found in all cortical layers [43,57]. 
Beyond individual tTF candidates, recent studies have used single-cell transcriptomics to investigate the temporal diversity and transcriptional dynamics of APs across neurogenesis $\left[32^{\bullet \bullet}, 45,46\right]$. In a recent study [46], we found that type-specific neuronal identity emerges from the apposition of generic differentiation programs onto ground state, embryonic age-dependent temporal identities. The coincidence between the initially shared temporal identity between newborn neurons and their mother progenitor is similar to how the progeny of Drosophila neuroblasts are temporally patterned by their mother cells (see Refs. [2,17]), with the difference that in the mammalian brain, interactions between multiple transcriptional programs rather than single tTFs appear to be at play.

Epigenetic regulation plays a critical role in the progression of neocortical progenitor identity. This has been the topic of a detailed recent review [62] and will only be briefly discussed here. A recent study analyzing the DNA methylation status of progenitors at different stages of corticogenesis reported that APs are regulated by three successive waves of demethylation, coinciding with the period of neurogenesis, astrogenesis and oligodendrogenesis $\left[63^{\circ}\right]$. In line with this, in neurogenic APs the promoters of core astrocytic genes are hypermethylated, preventing APs to respond to gliogenic extracellular cues that are already present early in corticogenesis [64-66]. Similarly, in late APs, the Polycomb group complex (which is also involved in identity progression in Drosophila, see Ref. [38] and discussion above) has been reported to repress the promoter of proneural genes such as $\mathrm{Ngn} 1$, thus favoring the transition to gliogenesis [67].

Non-cell autonomous controls over AP temporal identity As has been reported for Drosophila larval neurogenesis $\left[33^{\bullet \bullet}, 34\right]$, cell-extrinsic factors are involved in the progression of temporal identity in the mammalian neocortex (Figure 2c).

We have recently shown that progressive hyperpolarization is required for progression in the neurogenic competence of APs, through a mechanism involving regulation of Wnt signaling [68 ${ }^{\bullet \bullet}$ ]. Supporting a pathophysiological relevance of these findings, mutation in the sodium channel SGN3A, which is expressed in cortical progenitors, leads to cortical folding defects in humans [69 ${ }^{\circ}$. Thus, environmental signals, by regulating AP membrane potential, may affect the course of neurogenesis. Given their anatomical location lining the lateral ventricles, APs are directly influenced by the cerebrospinal fluid (CSF), which contains a large and highly dynamic set of diffusible proteins including regulators of cell survival and proliferation [70-73]. Neuron-derived signals may also be involved, and thalamic axons, in particular, may serve as a source of signaling factors to modulate the cell cycle length, proliferation rate and neuronal output of cortical progenitors and fine-tune post-mitotic neuronal identities in an area-specific manner [74-76].

Illustrating a role for neuronal progeny in controlling AP behavior, deletion of the transcription factor SIP1 specifically in newborn neurons leads to a precocious generation of superficial layer neurons and increased gliogenesis through feedback Fgf9, Ntf3 and Wnt signaling from newly born neurons to cortical progenitor cells $[77,78]$. In addition, the embryonic genetic ablation of deep layer neurons using Neurog $2^{\text {CreER/+ }}$ mice lengthens the period of deep layer neuron production at the expense of superficial layer generation, suggesting that feedback cues from post-mitotic deep layer neurons are transmitted to APs to allow their temporal transitions and generation of superficial layer neurons [79].

Finally, as mentioned above, post-mitotic controls over the temporal identity of neurons are also at play in the developing neocortex, including through interactions with subplate and thalamocortical afferents $\left[80^{\circ}, 81^{\circ}\right]$, which together sculpt developing neurons into their final stage-specific identity.

\section{Perspectives}

To sequentially generate distinct neuronal cell types, neuronal progenitors progressively change their temporal identity by integrating cell-autonomous transcriptional dynamics and environmental cues. This progression determines the identity of the neuronal progeny, and hence subsequent circuit assembly and function. Given the overarching role of temporal patterning in the assembly of the nervous system, it will be interesting to examine whether abnormal molecular patterning, once better characterized, is a common process at the root of seemingly disparate neurodevelopmental disorders.

\section{Conflict of interest statement}

Nothing declared.

\section{Acknowledgements}

Work in the Jabaudon laboratory is supported by the Swiss National Science Foundation and the Carigest Foundation. GA is supported by a PhD fellowship from the belgian F.N.R.S-F.R.I.A and by the Fonds Leon Fredericq.

\section{References and recommended reading}

Papers of particular interest, published within the period of review, have been highlighted as:

- of special interest

$\bullet$ of outstanding interest

1. Jeong H, Tiwari VK: Exploring the complexity of cortical development using single-cell transcriptomics. Front Neurosci $2018,12: 31$

2. Kohwi M, Doe CQ: Temporal fate specification and neural progenitor competence during development. Nat Rev Neurosci 2013, 14:823-838. 
3. Guillemot F: Spatial and temporal specification of neural fates by transcription factor codes. Development 2007, 134:37713780.

4. Dastjerdi FV, Consalez GG, Hawkes R: Pattern formation during development of the embryonic cerebellum. Front Neuroanat 2012, 6:10

5. Hu JS, Vogt D, Sandberg M, Rubenstein JL: Cortical interneuron development: a tale of time and space. Development 2017, 144:3867-3878.

6. Urbach R, Technau GM: Neuroblast formation and patterning during early brain development in Drosophila. Bioessays 2004 26:739-751.

7. Borello U, Pierani A: Patterning the cerebral cortex: traveling with morphogens. Curr Opin Genet Dev 2010, 20:408-415.

8. Azzarelli R, Hardwick LJA, Philpott A: Emergence of neuronal diversity from patterning of telencephalic progenitors. Wiley Interdiscip Rev Dev Biol 2015, 4:197-214.

9. Holguera I, Desplan C: Neuronal specification in space and time. Science 2018, 362:176-180.

10. Doe CQ: Temporal patterning in the Drosophila CNS. Annu Rev Cell Dev Biol 2017, 33:219-240.

11. Li X, Chen Z, Desplan C: Temporal patterning of neural progenitors in Drosophila. Curr Top Dev Biol 2013, 105:69-96.

12. Miyares RL, Lee T: Temporal control of Drosophila central nervous system development. Curr Opin Neurobiol 2018, 56:2432.

13. Skeath JB, Thor S: Genetic control of Drosophila nerve cord development. Curr Opin Neurobiol 2003, 13:8-15.

14. Truman JW, Bate M: Spatial and temporal patterns of neurogenesis in the central nervous system of Drosophila melanogaster. Dev Biol 1988, 125:145-157.

15. Prokop A, Technau GM: The origin of postembryonic neuroblasts in the ventral nerve cord of Drosophila melanogaster. Development 1991, 111:79-88.

16. Brody T, Odenwald WF: Programmed transformations in neuroblast gene expression during Drosophila CNS lineage development. Dev Biol 2000, 226:34-44.

17. Isshiki T, Pearson B, Holbrook S, Doe CQ: Drosophila neuroblasts sequentially express transcription factors which specify the temporal identity of their neuronal progeny. Cell 2001, 106:511-521.

18. Kambadur R, Koizumi K, Stivers C, Nagle J, Poole SJ, Odenwald WF: Regulation of POU genes by castor and hunchback establishes layered compartments in the Drosophila CNS. Genes Dev 1998, 12:246-260.

19. Jabaudon D: Fate and freedom in developing neocortical circuits. Nat Commun 2017, 8:16042.

20. Greig LC, Woodworth MB, Galazo MJ, Padmanabhan H, Macklis JD: Molecular logic of neocortical projection neuron specification, development and diversity. Nat Rev Neurosci 2013, 14:755-769.

21. Elliott J, Jolicoeur C, Ramamurthy V, Cayouette M: Ikaros confers early temporal competence to mouse retinal progenitor cells. Neuron 2008, 60:26-39.

22. Alsio JM, Tarchini B, Cayouette M, Livesey FJ: Ikaros promotes early-born neuronal fates in the cerebral cortex. Proc Natl Acad Sci U S A 2013, 110:E716-E725.

23. Mattar P, Ericson J, Blackshaw S, Cayouette M: A conserved regulatory logic controls temporal identity in mouse neural progenitors. Neuron 2015, 85:497-504.

24. Bello BC, Izergina N, Caussinus E, Reichert H: Amplification of neural stem cell proliferation by intermediate progenitor cells in Drosophila brain development. Neural Dev 2008, 3:5.

25. Boone JQ, Doe CQ: Identification of Drosophila type II neuroblast lineages containing transit amplifying ganglion mother cells. Dev Neurobiol 2008, 68:1185-1195.
26. Bayraktar OA, Doe CQ: Combinatorial temporal patterning in progenitors expands neural diversity. Nature 2013, 498:449455.

27. Grosskortenhaus R, Pearson BJ, Marusich A, Doe CQ: Regulation of temporal identity transitions in Drosophila neuroblasts. Dev Cell 2005, 8:193-202.

28. Kanai MI, Okabe M, Hiromi Y: Seven-up controls switching of transcription factors that specify temporal identities of Drosophila neuroblasts. Dev Cell 2005, 8:203-213.

29. Mettler U, Vogler G, Urban J: Timing of identity: spatiotempora regulation of hunchback in neuroblast lineages of Drosophila by Seven-up and prospero. Development 2006, 133:429-437.

30. Kohwi M, Hiebert LS, Doe CQ: The pipsqueak-domain proteins Distal antenna and Distal antenna-related restrict Hunchback neuroblast expression and early-born neuronal identity. Development 2011, 138:1727-1735.

31. Naka H, Nakamura S, Shimazaki T, Okano H: Requirement for COUP-TFI and II in the temporal specification of neural stem cells in CNS development. Nat Neurosci 2008, 11:1014-1023.

32. Okamoto M, Miyata T, Konno D, Ueda HR, Kasukawa T,

-• Hashimoto M, Matsuzaki F, Kawaguchi A: Cell-cycleindependent transitions in temporal identity of mammalian neural progenitor cells. Nat Commun 2016, 7:11349.

This study identified subsets of temporally regulated genes in cortical progenitors and showed that temporal transitions occur even in cell cycle arrested progenitors. It also showed that temporal transitions of in vitro cultured progenitors strongly depend on culture conditions, highlighting the importance of extrinsic feedback cues to temporal progression of progenitors.

33. Syed MH, Mark B, Doe CQ: Steroid hormone induction of -• temporal gene expression in Drosophila brain neuroblasts generates neuronal and glial diversity. eLife 2017, 6

This study identified novel tTFs in larval central brain neuroblasts and was the first to show that extrinsic signaling via ecdysone receptors is necessary for a major transition from early to late neuroblast temporal state.

34. Syed MH, Mark B, Doe CQ: Playing well with others: extrinsic cues regulate neural progenitor temporal identity to generate neuronal diversity. Trends Genet 2017, 33:933-942.

35. Pearson BJ, Doe CQ: Regulation of neuroblast competence in Drosophila. Nature 2003, 425:624-628.

36. Cleary MD, Doe CQ: Regulation of neuroblast competence: multiple temporal identity factors specify distinct neuronal fates within a single early competence window. Genes Dev 2006, 20:429-434.

37. Kohwi M, Lupton JR, Lai S-L, Miller MR, Doe CQ: Developmentally regulated subnuclear genome reorganization restricts neural progenitor competence in Drosophila. Cell 2013, 152:97-108.

38. Touma JJ, Weckerle FF, Cleary MD: Drosophila polycomb complexes restrict neuroblast competence to generate motoneurons. Development 2012, 139:657-666.

39. Pereira JD, Sansom SN, Smith J, Dobenecker M-W, Tarakhovsky A, Livesey FJ: Ezh2, the histone methyltransferase of PRC2, regulates the balance between self-renewal and differentiation in the cerebral cortex. Proc Natl Acad Sci U S A $2010,107: 15957-15962$

40. Morimoto-Suzki N, Hirabayashi Y, Tyssowski K, Shinga J, Vidal M, Koseki H, Gotoh Y: The polycomb component Ring1B regulates the timed termination of subcerebral projection neuron production during mouse neocortical development. Development 2014, 141:4343-4353.

41. Farnsworth DR, Bayraktar OA, Doe CQ: Aging neural progenitors lose competence to respond to mitogenic notch signaling. Curr Biol 2015, 25:3058-3068.

42. Gao P, Postiglione MP, Krieger TG, Hernandez L, Wang C, Han Z, Streicher C, Papusheva E, Insolera R, Chugh K et al.: Deterministic progenitor behavior and unitary production of neurons in the neocortex. Cell 2014, 159:775-788. 
43. Guo C, Eckler MJ, McKenna WL, McKinsey GL, Rubenstein JLR, Chen B: Fezf2 expression identifies a multipotent progenitor for neocortical projection neurons, astrocytes, and oligodendrocytes. Neuron 2013, 80:1167-1174.

44. Franco SJ, Gil-Sanz C, Martinez-Garay I, Espinosa A, HarkinsPerry SR, Ramos C, Müller U: Fate-restricted neural progenitors in the mammalian cerebral cortex. Science 2012, 337:746-749.

45. Yuzwa SA, Borrett MJ, Innes BT, Voronova A, Ketela T, Kaplan DR, Bader GD, Miller FD: Developmental emergence of adult neural stem cells as revealed by single-cell transcriptional profiling. Cell Rep 2017, 21:3970-3986.

46. Telley L, Agirman G, Prados J, Fievre S, Oberst P, Vitali I, Nguyen L, Dayer A, Jabaudon D: Single-cell transcriptional dynamics and origins of neuronal diversity in the developing mouse neocortex. bioRxiv 2018 http://dx.doi.org/10.1101/409458.

47. Gaspard N, Bouschet T, Hourez R, Dimidschstein J, Naeije G, van den Ameele J, Espuny-Camacho I, Herpoel A, Passante L, Schiffmann SN et al: : An intrinsic mechanism of corticogenesis from embryonic stem cells. Nature 2008, 455:351-357.

48. Espuny-Camacho I, Michelsen KA, Gall D, Linaro D, Hasche A, Bonnefont J, Bali C, Orduz D, Bilheu A, Herpoel A et al.: Pyramida neurons derived from human pluripotent stem cells integrate efficiently into mouse brain circuits in vivo. Neuron 2013, 77:440-456.

49. Camp JG, Badsha F, Florio M, Kanton S, Gerber T, WilschBräuninger M, Lewitus E, Sykes A, Hevers W, Lancaster M et al.: Human cerebral organoids recapitulate gene expression programs of fetal neocortex development. Proc Natl Acad Sci U $S$ A 2015, 112:15672-15677.

50. McConnell SK: Fates of visual cortical neurons in the ferret after isochronic and heterochronic transplantation. J Neurosci $1988,3: 945-974$

51. Mcconnell SK, Kaznowski CE: Cell cycle dependence of laminar determination in developing neocortex. Science 1991, 254:282 285.

52. Frantz GD, McConnell SK: Restriction of late cerebral cortical progenitors to an upper-layer fate. Neuron 1996, 17:55-61.

53. Oberst P, Fievre S, Baumann N, Concetti C, Jabaudon D: Apical progenitors remain multipotent throughout cortical neurogenesis. bioRxiv $2018 \mathrm{http}: / / \mathrm{dx}$.doi.org/10.1101/478891.

54. Hanashima C, Li SC, Shen L, Lai E, Fishell G: Foxg1 suppresses early cortical cell fate. Science 2004, 303:56-59.

55. Fame RM, MacDonald JL, Macklis JD: Development, specification, and diversity of callosal projection neurons. Trends Neurosci 2011, 34:41-50.

56. McKenna WL, Betancourt J, Larkin KA, Abrams B, Guo C, Rubenstein JLR, Chen B: Tbr1 and Fezf2 regulate alternate corticofugal neuronal identities during neocortical development. J Neurosci 2011, 31:549-564.

57. Chen B, Wang SS, Hattox AM, Rayburn H, Nelson SB, McConnell SK: The Fezf2-Ctip2 genetic pathway regulates the fate choice of subcortical projection neurons in the developing cerebral cortex. Proc Natl Acad Sci U S A 2008, 105:11382-11387.

58. Han W, Kwan KY, Shim S, Lam MMS, Shin Y, Xu X, Zhu Y, Li M, Sestan N: TBR1 directly represses Fezf2 to control the laminar origin and development of the corticospinal tract. Proc Nat Acad Sci U S A 2011, 108:3041-3046.

59. Srinivasan K, Leone DP, Bateson RK, Dobreva G, Kohwi Y, KohwiShigematsu T, Grosschedl R, McConnell SK: A network of genetic repression and derepression specifies projection fates in the developing neocortex. Proc Natl Acad Sci U S A 2012, 109:19071-19078.

60. Zahr SK, Yang G, Kazan H, Borrett MJ, Yuzwa SA, Voronova A,

- Kaplan DR, Miller FD: A translational repression complex in developing mammalian neural stem cells that regulates neuronal specification. Neuron 2018, 97:520-537.e6.

This study showed that apical progenitors are transcriptionally bimodal as they express identifiers of both deep and superficial layer neurons at the
mRNA level, but the translation of the inappropriate marker is inhibited by a translational repressive complex.

61. Azim E, Shnider SJ, Cederquist GY, Sohur US, Macklis JD: Lmo4 and Clim1 progressively delineate cortical projection neuron subtypes during development. Cereb Cortex 2009, 19:62-69.

62. Yoon K-J, Vissers C, Ming G-L, Song H: Epigenetics and epitranscriptomics in temporal patterning of cortical neural progenitor competence. J Cell Biol 2018, 217:1901-1914.

63. Sanosaka T, Imamura T, Hamazaki N, Chai M, Igarashi K, Ideta-

- Otsuka M, Miura F, Ito T, Fujii N, lkeo K et al:: DNA methylome analysis identifies transcription factor-based epigenomic signatures of multilineage competence in neural stem/ progenitor cells. Cell Rep 2017, 20:2992-3003.

This study reported the DNA methylation dynamics of cortical progenitors along corticogenesis and identified waves of demethylation coinciding with the neurogenic and gliogenic period.

64. Takizawa T, Nakashima K, Namihira M, Ochiai W, Uemura A Yanagisawa M, Fujita N, Nakao M, Taga T: DNA methylation is a critical cell-intrinsic determinant of astrocyte differentiation in the fetal brain. Dev Cell 2001, 1:749-758.

65. Fan G, Martinowich K, Chin MH, He F, Fouse SD, Hutnick L, Hattori D, Ge W, Shen Y, Wu H et al:: DNA methylation controls the timing of astrogliogenesis through regulation of JAK-STAT signaling. Development 2005, 132:3345-3356.

66. He F, Ge W, Martinowich K, Becker-Catania S, Coskun V, Zhu W, Wu H, Castro D, Guillemot F, Fan G et al.: A positive autoregulatory loop of Jak-STAT signaling controls the onset of astrogliogenesis. Nat Neurosci 2005, 8:616-625.

67. Hirabayashi Y, Suzki N, Tsuboi M, Endo TA, Toyoda T, Shinga J, Koseki H, Vidal M, Gotoh Y: Polycomb limits the neurogenic competence of neural precursor cells to promote astrogenic fate transition. Neuron 2009, 63:600-613.

68. Vitali I, Fièvre S, Telley L, Oberst P, Bariselli S, Frangeul L,

- Baumann N, McMahon JJ, Klingler E, Bocchi R et al.: Progenitor hyperpolarization regulates the sequential generation of neuronal subtypes in the developing neocortex. Cell 2018, 174:1264-1276.e15

This study showed that bioelectric membrane properties are permissive for temporal progression of cortical apical progenitors and that hyperpolarization leads to a forward shift in apical progenitor temporal state via inhibition of Wnt-beta-catenin signaling.

69. Smith RS, Kenny CJ, Ganesh V, Jang A, Borges-Monroy R,

- $\quad$ Partlow JN, Hill RS, Shin T, Chen AY, Doan RN et al.: Sodium channel SCN3A (NaV1.3) regulation of human cerebral cortical folding and oral motor development. Neuron 2018, 99:905-913. e7.

This study showed that the voltage-gated sodium channel $\mathrm{Nav}_{1} 1.3$ plays an important role in the prenatal development of human cortical areas and that mutations in $\mathrm{Na}_{v} 1.3$ disrupts cortical folding

70. Zappaterra MD, Lisgo SN, Lindsay S, Gygi SP, Walsh CA, Ballif BA A comparative proteomic analysis of human and rat embryonic cerebrospinal fluid. J Proteome Res 2007, 6:35373548 .

71. Lehtinen MK, Zappaterra MW, Chen X, Yang YJ, Hill AD, Lun M, Maynard T, Gonzalez D, Kim S, Ye P et al: The cerebrospinal fluid provides a proliferative niche for neural progenitor cells. Neuron 2011, 69:893-905.

72. Lehtinen MK, Walsh CA: Neurogenesis at the braincerebrospinal fluid interface. Annu Rev Cell Dev Biol 2011 27:653-679.

73. Chau KF, Springel MW, Broadbelt KG, Park H-Y, Topal S, Lun MP Mullan $\mathrm{H}$, Maynard T, Steen $\mathrm{H}$, LaMantia AS et al: Progressive differentiation and instructive capacities of amniotic fluid and cerebrospinal fluid proteomes following neural tube closure. Dev Cell 2015, 35:789-802.

74. Pouchelon G, Frangeul L, Rijli FM, Jabaudon D: Patterning of prethalamic somatosensory pathways. Eur J Neurosci 2012 35:1533-1539.

75. Vue TY, Lee M, Tan YE, Werkhoven Z, Wang L, Nakagawa Y: Thalamic control of neocortical area formation in mice. $J$ Neurosci 2013, 33:8442-8453. 
76. Dehay C, Savatier P, Cortay V, Kennedy H: Cell-cycle kinetics of neocortical precursors are influenced by embryonic thalamic axons. J Neurosci 2001, 21:201-214.

77. Seuntjens E, Nityanandam A, Miquelajauregui A, Debruyn J, Stryjewska A, Goebbels S, Nave K-A, Huylebroeck D, Tarabykin V: Sip1 regulates sequential fate decisions by feedback signaling from postmitotic neurons to progenitors. Nat Neurosci 2009 , 12:1373-1380.

78. Parthasarathy S, Srivatsa S, Nityanandam A, Tarabykin V: Ntf3 acts downstream of Sip1 in cortical postmitotic neurons to control progenitor cell fate through feedback signaling. Development 2014, 141:3324-3330.

79. Toma K, Kumamoto T, Hanashima C: The timing of upper-layer neurogenesis is conferred by sequential derepression and negative feedback from deep-layer neurons. J Neurosci 2014 , 34:13259-13276.

80. Ozair MZ, Kirst C, van den Berg BL, Ruzo A, Rito T, Brivanlou AH: hPSC

- modeling reveals that fate selection of cortical deep projection neurons occurs in the subplate. Cell Stem Cell 2018, 23:60-73.e6.

This study highlights the importance of the transient expansion of the subplate in primate corticogenesis as subplate neurons can be modulated postmitotically by Wnt-signaling to specify into all classes of deep layer projecting neurons.

81. Ohtaka-Maruyama C, Okamoto M, Endo K, Oshima M, Kaneko N,

- Yura K, Okado H, Miyata T, Maeda N: Synaptic transmission from subplate neurons controls radial migration of neocortical neurons. Science 2018, 360:313-317.

This study showed that in the developing cortex, subplate neurons develop transient synapses to contact migrating neurons and instruct their multipolar to bipolar morphological transition. 